\title{
Thiamine as an adjunctive therapy in cardiac surgery: a randomized, double- blind, placebo-controlled, phase II trial
}

Lars W. Andersen ${ }^{1,2,3^{*}}$, Mathias J. Holmberg ${ }^{1,3}$, Katherine M. Berg ${ }^{4}$, Maureen Chase ${ }^{1}$, Michael N. Cocchi ${ }^{1,5}$, Christopher Sulmonte', Julia Balkema', Mary MacDonald', Sophia Montissol', Venkatachalam Senthilnathan', David Liư ${ }^{6}$, Kamal Khabbaz ${ }^{6}$, Adam Lerner ${ }^{7}$, Victor Novack ${ }^{8,9}$, Xiaowen Liu and Michael W. Donnino ${ }^{1,4}$

\begin{abstract}
Background: Thiamine is a vitamin that is essential for adequate aerobic metabolism. The objective of this study was to determine if thiamine administration prior to coronary artery bypass grafting would decrease post-operative lactate levels as a measure of increased aerobic metabolism.

Methods: We performed a randomized, double-blind, placebo-controlled trial of patients undergoing coronary artery bypass grafting. Patients were randomized to receive either intravenous thiamine $(200 \mathrm{mg})$ or placebo both immediately before and again after the surgery. Our primary endpoint was post-operative lactate levels. Additional endpoints included pyruvate dehydrogenase activity, global and cellular oxygen consumption, post-operative complications, and hospital and intensive care unit length of stay.

Results: Sixty-four patients were included. Thiamine levels were significantly higher in the thiamine group as compared to the placebo group immediately after surgery $(1200[683,1200] \mathrm{nmol} / \mathrm{L}$ vs. $9[8,13] \mathrm{nmol} / \mathrm{L}, p<0.001)$. There was no difference between the groups in the primary endpoint of lactate levels immediately after the surgery $(2.0[1.5,2.6] \mathrm{mmol} / \mathrm{L}$ vs. $2.0[1.7,2.4], p=0.75)$. Relative pyruvate dehydrogenase activity was lower immediately after the surgery in the thiamine group as compared to the placebo group (15\% [11, 37] vs. $28 \%$ $[15,84], p=0.02)$. Patients receiving thiamine had higher post-operative global oxygen consumption 1 hour after the surgery (difference: $0.37 \mathrm{~mL} / \mathrm{min} / \mathrm{kg}$ [95 \% Cl: $0.03,0.71], p=0.03$ ) as well as cellular oxygen consumption. We found no differences in clinical outcomes.
\end{abstract}

Conclusions: There were no differences in post-operative lactate levels or clinical outcomes between patients receiving thiamine or placebo. Post-operative oxygen consumption was significantly increased among patients receiving thiamine.

Trial registration: clinicaltrials.gov NCT02322892, December 14, 2014

Keywords: Thiamine, Lactate, Cardiac surgery, Coronary artery bypass grafting, Pyruvate dehydrogenase, Aerobic, Anaerobic, Metabolism, Oxygen consumption

\footnotetext{
*Correspondence: Iwanders@bidmc.harvard.edu

${ }^{1}$ Center for Resuscitation Science, Department of Emergency Medicine, Beth Israel Deaconess Medical Center, 330 Brookline Avenue, Boston, MA 02215, USA

2Department of Anesthesiology, Aarhus University Hospital, Nørrebrogade 44, 8000 Aarhus C, Denmark

Full list of author information is available at the end of the article
} 


\section{Background}

Over 230,000 patients in the United States undergo coronary artery bypass grafting (CABG) each year [1]. Although mortality in this patient population is relatively low, complications increasing both morbidity and length of stay remain significant [2-4]. Lactate elevation, a marker of anaerobic metabolism, is commonly seen after major cardiac surgery and multiple studies have found an association between elevated post-operative lactate levels and increased morbidity and mortality [5-10]. Elevated lactate has also been associated with poor outcome in other forms of critical illness, such as sepsis [11, 12]. The rise in lactate in these settings is thought to be secondary to inadequate oxygen delivery and/or a deficit in aerobic metabolism [13]. The importance of this decrease in aerobic metabolism is also supported by an association between low oxygen consumption and poor outcome in critically ill states including sepsis, high-risk surgery, and post-cardiac arrest patients [14-16].

Aerobic metabolism occurs when pyruvate enters the mitochondria through pyruvate decarboxylation to acetylcoenzyme A. This entry into the mitochondria (and tricarboxylic acid [TCA] cycle) is facilitated by the ratelimiting enzyme pyruvate dehydrogenase (PDH) (Fig. 1) $[17,18]$. Decreased PDH activity may cause a shift toward

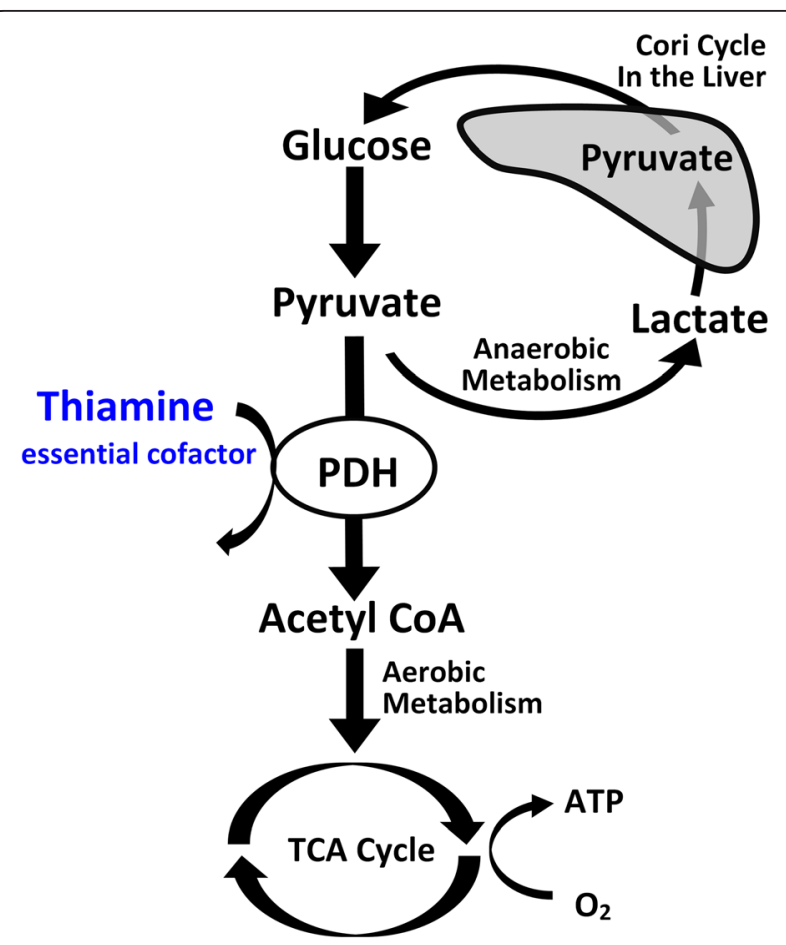

Fig. 1 Simplified graphical presentation of PDH's and thiamine's role in aerobic metabolism. Aerobic metabolism occurs when pyruvate enters the mitochondria through pyruvate decarboxylation to acetyl-coenzyme A, facilitated by the rate-limiting enzyme pyruvate dehydrogenase $(\mathrm{PDH})$. Adapted with permission from Andersen et al. [12]. ATP adenosine triphosphate, CoA coenzyme A, TCA tricarboxylic acid anaerobic metabolism and potentially play a role in the metabolic changes (i.e., elevated lactate) seen in patients undergoing CABG with cardiopulmonary bypass [19-24]. Thiamine (vitamin B1) is an essential co-factor for $\mathrm{PDH}$ function. In the absence of thiamine, the conversion of pyruvate to acetyl-coenzyme A is inhibited, cellular oxygen consumption is decreased, and lactate is produced [25]. Thiamine deficiency is common after cardiac surgery and post-operative thiamine levels have been found to be inversely associated with post-operative lactate levels $[19,26]$.

We hypothesized that thiamine administration prior to surgery would decrease post-operative lactate levels in patients undergoing CABG by increasing PDH activity and oxygen consumption, ultimately leading to improved clinical outcomes.

\section{Methods}

Design and setting

This was a single-center, randomized, double-blind, placebo-controlled, phase II trial of thiamine in patients undergoing CABG with cardiopulmonary bypass. Patients were enrolled at Beth Israel Deaconess Medical Center, Boston, MA, USA - a tertiary care center with more than 450 CABG surgeries performed annually. The study was approved by The Committee on Clinical Investigations at Beth Israel Deaconess Medical Center (protocol number: 2014-P000257) and patients provided written informed consent prior to enrollment. The trial was registered at clinicaltrials.gov (NCT02322892) and funded by the American Heart Association (15CRP22830000).

\section{Study population}

We enrolled consecutive patients between January 2015 and July 2015. We included adult patients ( $\geq 21$ years) scheduled for CABG with or without concomitant valve procedures. Due to unpublished preliminary data suggesting that patients with a higher European System for Cardiac Operative Risk Evaluation (EuroSCORE) II score have higher post-operative lactate levels, we only included patients with a EuroSCORE II score $>1.5 \%$. We excluded patients based on the following criteria: (1) current thiamine supplementation or known clinical indication for thiamine (e.g., alcohol dependency), (2) known allergy to thiamine, (3) emergent or salvage CABG (as defined by the Society of Thoracic Surgeons [27]), (4) CABG without cardiopulmonary bypass ("off-pump" surgery), and (5) protected population (pregnant women, prisoners, and the intellectually disabled).

\section{Randomization, study drug and blinding}

Patients were randomized in blocks of four in a 1:1 ratio to receive thiamine or placebo. The randomization was stratified by "high" (>4 \%) vs. "low" ( $\leq 4 \%$ ) EuroSCORE 
II to minimize baseline heterogeneity between the two groups. Patients received $200 \mathrm{mg}$ thiamine in $50 \mathrm{~mL} 0.9 \%$ saline or matching placebo (50 mL $0.9 \%$ saline) immediately before the surgery and again immediately after the surgery upon arrival in the intensive care unit. The placebo and the thiamine were identical in appearance; patients, healthcare personnel, and the research team remained blinded throughout the study period.

\section{Endpoints and data collection}

The primary endpoint was post-operative lactate levels collected upon arrival to the intensive care unit. Key secondary endpoints included PDH activity, post-operative complications, intensive care unit and hospital length of stay, and mortality. Additional endpoints included lactate levels 6 hours after the surgery, time on mechanical ventilation (defined as the time from the end of surgery until extubation), time on vasopressors (defined as the time from the end of surgery until discontinuation of all vasopressors [epinephrine, norepinephrine, phenylephrine, dopamine, and vasopressin] for at least 6 hours), and cellular and global oxygen consumption (see below). Post-operative complications before hospital discharge included: new atrial fibrillation (requiring treatment or lasting for $>24$ hours), renal failure (requiring new dialysis), stroke as defined by Sacco et al. [28], myocardial infarction using the universal definition [29], acute respiratory distress syndrome using the Berlin definition [30], infection (requiring new antibiotics), and documented delirium.

Before the surgery, we recorded demographic data and past medical history including the New York Heart Association (NYHA) classification [31] and the Canadian Cardiovascular Society grading of angina pectoris [32]. We also calculated the EuroSCORE II [33], which is a validated pre-operative score to predict post-operative morbidity and mortality [34-36]. The score utilizes information regarding the patient (age, gender, and co-morbidities) and current cardiac status (NYHA class, presence of unstable angina, left ventricular function and more), as well as factors related to the operation (urgency and type of intervention).

All data were collected by a trained research assistant according to a detailed, pre-defined data dictionary and all outcome variables were verified by a physician. Data were entered into a secure, online database (Research Electronic Data Capture [RedCAP]) [37].

\section{Blood samples}

Blood samples were obtained immediately before administration of the first study dose, upon arrival to the intensive care unit (immediately before administration of the second study dose), and again 6 hours later. Upon collection, blood was sent to the hospital's clinical laboratory for lactate measurements (Rapidlab 1265, Siemens Healthcare Diagnostics Inc., Tarrytown, NY, USA). Fresh whole blood collected in ethylenediaminetetraacetic acid tubes was used for measurement of PDH and cellular oxygen consumption (see below). The remaining blood was centrifuged at $3500 \mathrm{rpm}$ for 10 minutes. Plasma and serum were aliquoted into light-protected cryotubes and frozen at $-80{ }^{\circ} \mathrm{C}$ for later measurements of thiamine levels. All blood samples were collected from pre-existing arterial lines except one draw that was collected from a preexisting central venous line.

\section{Thiamine levels and pyruvate dehydrogenase}

Thiamine levels were measured in plasma via liquid chromatography-tandem mass spectrometry by Quest Diagnostics (Nichols Institute, Chantilly, VA, USA). Absolute thiamine deficiency was determined using a previously established standard laboratory reference range from Quest Diagnostics; specifically, absolute thiamine deficiency was defined as a level $\leq 7 \mathrm{nmol} / \mathrm{L}$. If a thiamine level was undetectable (i.e., $<7 \mathrm{nmol} / \mathrm{L}$ ) a value of $7 \mathrm{nmol} /$ L was imputed.

Peripheral blood mononuclear cells (PBMCs) were isolated from fresh whole blood using a density gradient separation method (Ficoll-Paque premium, GE Healthcare Bio-Science Corp., Piscataway, NJ, USA). PDH activity and quantity were then measured after disruption of the mitochondrial membrane via an immunocapture and microplate-based assay as previously described [38, 39]. PDH specific activity was calculated as PDH activity/ln (PDH quantity). PDH activity and quantity are expressed in $\mathrm{OD} / \mathrm{min} / \mathrm{mg}$ protein where OD indicates the absorbance (optical density). Post-operative PDH values are expressed as relative to the pre-operative $\mathrm{PDH}$ value (i.e., $\left[\mathrm{PDH}_{\text {post-surgery }} / \mathrm{PDH}_{\text {pre-surgery }}\right] \times 100 \%$ ).

\section{Global and cellular oxygen consumption}

Based on equipment availability, we measured global oxygen consumption $\left(\mathrm{VO}_{2}\right)$ in a subset of patients using a compact anesthesia monitor, which was connected to the ventilator tubing via a ventilator adapter with an attached gas sampling line (General Electric, Fairfield, CT, USA). This device measures $\mathrm{VO}_{2}$ continuously on a breath-by-breath basis using an incorporated pneumotachograph to measure the volume of gas being exchanged, and a paramagnetic analyzer to detect differences in inspired and expired oxygen [40, 41]. The monitor does not measure $\mathrm{VO}_{2}$ when the fraction of inspired oxygen is $>85 \%$. $\mathrm{VO}_{2}$ was recorded every 5 minutes from arrival in the intensive care unit until extubation or until the second post-operative blood draw. For the analysis, we used data from 1 hour after the surgery until 4 hours after the surgery. We removed non-physiological outliers $\left(\mathrm{VO}_{2}<150 \mathrm{~mL} / \mathrm{min}\right.$ and $\left.\mathrm{VO}_{2}>700 \mathrm{~mL} / \mathrm{min}\right)$ and very inconsistent values (i.e., single values that changed 
substantially within a short timeframe). $\mathrm{VO}_{2}$ was normalized to body weight.

Based on laboratory personnel and equipment availability (as the measurement must be performed at the time of sample collection), we measured the cellular oxygen consumption rate (OCR) in PBMCs on a subset of the enrolled patients. The complete mitochondrial respiration profile was measured using the XF Cell Mito Stress Test Kit in an $\mathrm{XF}^{\mathrm{e}} 96$ Extracellular Flux Analyzer (Seahorse Bioscience, North Billerica, MA, USA). This technology has been described in detail elsewhere [42]. For this manuscript, we report basal cellular respiration as well as maximal cellular respiration (see Additional file 1). The value of interest was the immediate post-surgery OCR relative to the pre-surgery OCR (i.e., $\left[\mathrm{OCR}_{\text {post-surgery }} /\right.$ $\left.\mathrm{OCR}_{\text {pre-surgery }}\right] \times 100 \%$ ).

\section{Sample size calculation and statistical analysis}

The sample size for the current study was based on unpublished preliminary data from a small open-label trial of thiamine administration and historical observational data (see Additional file 1). Based on this, we estimated that the placebo group would have a mean post-operative lactate level of $3.6 \mathrm{mmol} / \mathrm{L}$ and that the thiamine group would have a mean lactate level of $2.6 \mathrm{mmol} / \mathrm{L}$ with both groups having a standard deviation of $1.4 \mathrm{mmol} / \mathrm{L}$. Based on these estimates, 32 patients in each group provide $80 \%$ power for a two-sided $t$ test at an alpha level of 0.05 .

Descriptive statistics were used to characterize the study population; continuous variables are presented as means with standard deviations or medians with $1^{\text {st }}$ and $3^{\text {rd }}$ quartiles depending on the normality of the data. Categorical variables are presented as counts with relative frequencies. Continuous data were compared between the groups using a two-sample $t$ test or Wilcoxon rank sum test depending on normality of the data. Categorical data were compared between groups using Fisher's exact test. The primary analysis was a comparison of lactate levels immediately after the surgery. Secondarily, we used repeated measures analysis to analyze lactate levels immediately and 6 hours after surgery using an unstructured variancecovariance structure adjusting for the pre-surgery level as well as the stratification factor (i.e., low vs. high EuroSCORE II) [43]. PDH levels and $\mathrm{VO}_{2}$ were similarly analyzed using a repeated measures approach. For the latter analysis, we assumed a $1^{\text {st }}$ order autoregressive variancecovariance structure. OCR was compared between groups using linear regression with adjustment for the stratification factor. Right-skewed variables (lactate, PDH values and OCR) were log-transformed before analysis.

We performed three subgroup analyses for the primary endpoint; according to the stratification variable, according to the pre-surgery thiamine level (dichotomized as above/ below the median) and according to whether the patient had diabetes or not as a relatively large proportion of patients with diabetes may have thiamine deficiency $[44,45]$.

Analyses were conducted on a modified intention-totreat basis including only those subjects who received the first dose of the study drug [46]. Data were complete for the primary endpoint and no imputations were done for missing secondary outcomes. All statistical analyses were pre-defined (i.e., planned before unblinding of the data) unless otherwise specified. All hypothesis tests were two-sided, with a significance level of $p<0.05$. Given the pilot nature of the current study, no adjustments were made for multiple testing and all secondary outcomes should therefore be considered exploratory. Statistical analyses were conducted with the use of SAS software, version 9.4 (SAS Institute Inc., Cary, NC, USA).

\section{Results}

Study population and thiamine levels

Of 275 patients screened, 69 were randomized and 64 received the first study dose and were therefore included in the analysis (Fig. 2). One patient was scheduled for CABG and valve surgery but only received valve surgery. Per our pre-defined analysis plan, this patient was not excluded. The baseline characteristics of the patients are provided in Table 1 and surgical characteristics in Table 2. There were no statistically significant differences in surgical characteristics between the groups. There was no difference in the pre-operative lactate levels between the groups $(1.2 \mathrm{mmol} / \mathrm{L}[1.0,1.3]$ vs. $1.1 \mathrm{mmol} / \mathrm{L}[1.0,1.3])$.

Thiamine levels were similar between the thiamine and placebo group prior to the surgery $(14 \mathrm{nmol} / \mathrm{L}[11,18]$ vs. $14 \mathrm{nmol} / \mathrm{L}[12,18])$ with one patient in the thiamine group and zero patients in the placebo group having thiamine deficiency. Thiamine levels were substantially higher in the thiamine group as compared to the placebo group immediately after surgery $(1200 \mathrm{nmol} / \mathrm{L}[683,1200]$ vs. $9 \mathrm{nmol} / \mathrm{L}[8,13], p<0.001)$ and 6 hours after the surgery $(1200 \mathrm{nmol} / \mathrm{L}[872,1200]$ vs. $10 \mathrm{nmol} / \mathrm{L}[7,15]$, $p<0.001)$. No patients in the thiamine group were deficient after the surgery as compared to three $(9 \%)$ in the placebo group immediately after the surgery $(\mathrm{p}=0.24)$ and five $(15 \%)$ patients 6 hours after the surgery $(p=0.05)$.

\section{Lactate and PDH values}

There was no difference between the thiamine and placebo groups in the primary endpoint of lactate levels immediately after the surgery $(2.0 \mathrm{mmol} / \mathrm{L}[1.5,2.6]$ vs. $2.0 \mathrm{mmol} / \mathrm{L}$ $[1.7,2.4], p=0.75$, Fig. 3). There was no difference in lactate levels 6 hours after the surgery $(1.8 \mathrm{mmol} / \mathrm{L}[1.3,2.1] \mathrm{vs}$. $1.8 \mathrm{mmol} / \mathrm{L}[1.4,2.6], p=0.45$, Fig. 3). There was no difference in post-operative lactate levels when analyzed in the repeated measures model $(p=0.76)$.

The PDH values are presented in Fig. 4. Relative PDH activity was significantly lower immediately after the 


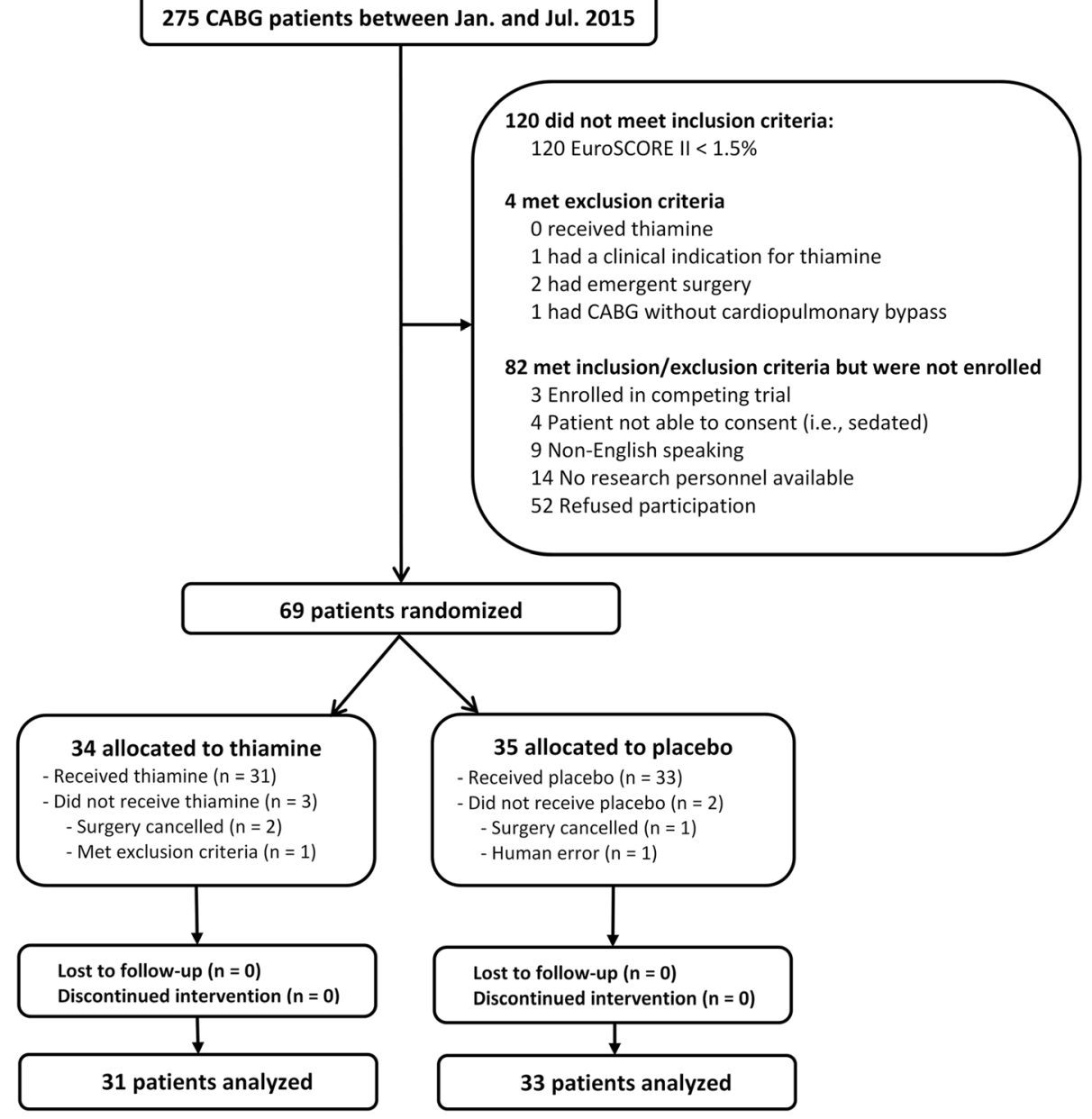

Fig. 2 CONSORT diagram. Out of 275 patients screened, 69 were randomized and 64 were analyzed per the modified intention-to-treat analysis. No patients discontinued the intervention or were lost to follow-up. CABG coronary artery bypass grafting, EuroSCORE European System for Cardiac Operative Risk Evaluation

surgery in the thiamine group as compared to the placebo group $(15 \%[11,37]$ vs. $28 \%[15,84], p=0.02)$. There was no difference 6 hours after the surgery $(23 \%$ $[9,50]$ vs. $29 \%[12,78], p=0.19)$. There was no difference in relative PDH quantity immediately after the surgery $(43 \%[22,75]$ vs. $50 \%[15,96], p=0.93)$ or 6 hours after the surgery $(56 \%[23,75]$ vs. $74 \%[24,111], p=$ 0.27 ). Relative PDH specific activity was lower in the thiamine group immediately after surgery $(19 \%[14,42]$ vs. $39 \%[20,87], p=0.01)$, but there was no difference 6 hours after surgery $(27 \%[12,59]$ vs. $41 \%[14,77], p=$ $0.23)$. Given some imbalance between groups in PDH activity at baseline, we performed a post hoc analysis including the baseline PDH activity in the model. In this model, the difference between the two groups in relative PDH activity immediately after the surgery was not significant $(p=0.12)$. A post hoc comparison of absolute PDH values are presented in Additional file 1. There was no group difference between any of the absolute PDH values.

\section{Oxygen consumption}

Global oxygen consumption $\left(\mathrm{VO}_{2}\right)$ was measured on 27 patients: 15 in the placebo group and 12 in the thiamine group. There was no significant difference in baseline characteristics between those who had and those who did not have $\mathrm{VO}_{2}$ measured (see Additional file 1). There was a significant difference in $\mathrm{VO}_{2} 1$ hour after surgery with the thiamine group having higher values (difference: $0.37 \mathrm{~mL} / \mathrm{min} / \mathrm{kg}$ [95 \% CI: 0.03, 0.71], $p=0.03$ ). There was no difference between the groups in change in $\mathrm{VO}_{2}$ over time from 1 to 4 hours after the surgery $(p=0.36)$. When the non-significant interaction was removed from the model, the between-group difference remained (difference: $0.19 \mathrm{~mL} / \mathrm{min} / \mathrm{kg}$ [95 \% CI: 0.05, 0.33], $p=0.01$ ) indicating that patients in the thiamine group had consistently higher $\mathrm{VO}_{2}$ values from one to four hours after the surgery.

Basal cellular oxygen consumption was available on 40 patients (20 in the placebo group and 20 in the thiamine 
Table 1 Baseline characteristics of the study patients ${ }^{a}$

\begin{tabular}{|c|c|c|}
\hline & Thiamine $(n=31)$ & Placebo $(n=33)$ \\
\hline \multicolumn{3}{|l|}{ Demographics } \\
\hline Age (years) & $71(67,75)$ & $73(68,79)$ \\
\hline Sex (female) & $6(19)$ & $10(30)$ \\
\hline Body mass index $\left(\mathrm{kg} / \mathrm{m}^{2}\right)$ & $29(26,33)$ & $28(25,29)$ \\
\hline \multicolumn{3}{|l|}{ Race } \\
\hline White & $31(100)$ & $32(97)$ \\
\hline Black & $0(0)$ & $1(3)$ \\
\hline EuroSCORE II (\%) & $3.4(2.1,5.2)$ & $2.3(1.9,4.5)$ \\
\hline EuroSCORE II > $4.0 \%$ & $9(29)$ & $12(36)$ \\
\hline \multicolumn{3}{|l|}{ Cardiac past medical history } \\
\hline $\mathrm{Ml} / \mathrm{PCl}$ & $14(45)$ & $12(36)$ \\
\hline Atrial fibrillation & $7(23)$ & $5(15)$ \\
\hline Previous cardiac surgery & $0(0)$ & $2(6)$ \\
\hline Chronic heart failure & $4(13)$ & $8(24)$ \\
\hline Valve disease & $12(39)$ & $8(24)$ \\
\hline Ejection fraction (\%) & $53(40,60)$ & $55(45,60)$ \\
\hline \multicolumn{3}{|l|}{ Current NYHA class $^{\mathrm{b}}$} \\
\hline । & $5(16)$ & $1(3)$ \\
\hline$\|$ & $12(29)$ & $14(42)$ \\
\hline III & $14(45)$ & $15(45)$ \\
\hline IV & $0(0)$ & $3(9)$ \\
\hline \multicolumn{3}{|l|}{ Current angina class $^{\mathrm{b}}$} \\
\hline No symptoms & $13(42)$ & $14(42)$ \\
\hline I & $0(0)$ & $1(3)$ \\
\hline$\|$ & $3(10)$ & $5(15)$ \\
\hline III & $7(23)$ & $7(21)$ \\
\hline IV & $8(26)$ & $8(18)$ \\
\hline \multicolumn{3}{|l|}{ Other past medical history } \\
\hline Pulmonary disease & $3(10)$ & $5(15)$ \\
\hline Diabetes & $14(45)$ & $14(42)$ \\
\hline Insulin dependent & $7(50)$ & $6(43)$ \\
\hline Non-insulin dependent & $7(50)$ & $8(57)$ \\
\hline Renal disease & $7(23)$ & $11(33)$ \\
\hline Cancer & $1(3)$ & $2(6)$ \\
\hline \multicolumn{3}{|l|}{ Pre-operative laboratory values } \\
\hline White blood count $\left(\times 10^{3}\right)$ & $8.2(6.8,8.9)$ & $7.5(5.7,10.0)$ \\
\hline Hemoglobin (g/dL) & $12.9(11.5,14.4)$ & $13.0(11.2,14.2)$ \\
\hline Creatinine (mg/dL) & $1.1(0.8,1.6)$ & $1.1(0.8,1.3)$ \\
\hline Glucose (mg/dL) & $131(108,164)$ & $114(94,145)$ \\
\hline \multicolumn{3}{|l|}{ Pre-surgical characteristics } \\
\hline \multicolumn{3}{|l|}{ Status } \\
\hline Elective & $14(45)$ & $12(36)$ \\
\hline Urgent & $17(55)$ & $21(65)$ \\
\hline
\end{tabular}

Table 1 Baseline characteristics of the study patients ${ }^{a}$ (Continued)

\begin{tabular}{lll} 
Location prior to surgery & & \\
Home & $13(42)$ & $9(27)$ \\
Ward & $15(48)$ & $24(73)$ \\
Intensive care unit & $3(10)$ & $0(0)$ \\
\hline
\end{tabular}

Abbreviations: EuroSCORE European System for Cardiac Operative Risk Evaluation, $\mathrm{MI}$ myocardial infarction, $\mathrm{PCl}$ percutaneous coronary intervention, NYHA New York Heart Association

${ }^{\text {a }}$ Categorical variables are presented as count (frequency) and continuous

variables as median (quartiles)

${ }^{b}$ Defined as the worst classification within the last 2 weeks

group). There was no significant difference in baseline characteristics between those who had and those who did not have cellular oxygen consumption measured except for a slightly higher EuroSCORE II in those without cellular oxygen consumption measured (see Additional file 1). We found a significant difference in post-operative relative basal oxygen consumption between the thiamine and placebo group (99\% $[89,126]$, vs. $85 \%[66,136] p=0.04$, Fig. 5). Maximal cellular oxygen consumption was available on 38 patients (19 in the placebo group and 19 in the thiamine group). We found a significant difference in post-operative relative maximal oxygen consumption between the thiamine and placebo group $(107 \%[86,155]$, vs. $90 \%[54,125], p=0.02$, Fig. 5).

\section{Clinical outcomes}

There was no difference in time to extubation (8.3 hours $[5.0,19.2]$ vs. 7.4 hours $[5.5,12.3], p=0.51)$ or time on vasopressors $(12.8$ hours $[4.0,21.1]$ vs. 9.3 hours [2.1, 22.1], $p=0.70$ ) between the thiamine and placebo group. There was no difference in intensive care unit length of stay $(2.4$ days $[1.1,4.2]$ vs. 2.3 days $[1.4,4.0], p=0.66)$ or in hospital length of stay (5 days $[4,8]$ vs. 5 days $[4,7]$, $p=0.49$ ). There was no difference between the two groups in post-operative complications (see Table 3). One patient died in the placebo group and none in the thiamine group. No side effects of the study medication were reported in either group.

\section{Subgroups analyses}

Twenty-eight patients had a pre-surgery thiamine level below $14 \mathrm{mmol} / \mathrm{L}$ (the median of the entire cohort); 14 in each group. In this subgroup, there was no difference in the post-operative lactate level between those receiving thiamine and placebo $(1.7 \mathrm{mmol} / \mathrm{L}[1.5,2.4]$ vs. $2.1 \mathrm{mmol} / \mathrm{L}[1.6,2.6], p=0.32)$. Twenty-one patients had a EuroSCORE II above $4.0 \%$; 12 in the placebo group and nine in the thiamine group. There was no difference between the groups in post-operative lactate $(3.0 \mathrm{mmol} / \mathrm{L}$ $[2.1,4.0]$ vs. $2.3 \mathrm{mmol} / \mathrm{L}[1.9,3.5], p=0.42)$ in this subgroup. Twenty-eight patients had diabetes; 14 in each group. There was no difference in post-operative 
Table 2 Surgical characteristics of the study patients ${ }^{a}$

\begin{tabular}{|c|c|c|c|}
\hline & $\begin{array}{l}\text { Thiamine } \\
(\mathrm{n}=31)\end{array}$ & $\begin{array}{l}\text { Placebo } \\
(n=33)\end{array}$ & $p$ value \\
\hline Vessels grafted & & & 0.14 \\
\hline 1 & $5(17)$ & $6(18)$ & \\
\hline 2 & $0(0)$ & $5(15)$ & \\
\hline 3 & $13(43)$ & $14(42)$ & \\
\hline 4 & $11(37)$ & $8(24)$ & \\
\hline 5 & $1(3)$ & $0(0)$ & \\
\hline Valve surgery & & & 0.15 \\
\hline None & $17(55)$ & $22(67)$ & \\
\hline Aortic & $13(42)$ & $7(21)$ & \\
\hline Mitral & $1(3)$ & $4(12)$ & \\
\hline Other procedure & $2(6)$ & $1(3)$ & 0.61 \\
\hline $\begin{array}{l}\text { Intra-operative } \\
\text { complication(s) }\end{array}$ & $2(6)$ & $0(0)$ & 0.23 \\
\hline Length of surgery (min) & $211(186,251)$ & $210(191,226)$ & 0.56 \\
\hline Bypass time (min) & $95(69,118)$ & $80(72,96)$ & 0.24 \\
\hline Cross-clamp time (min) & $77(54,98)$ & $63(54,76)$ & 0.13 \\
\hline Received red blood cells & $9(29)$ & $5(15)$ & 0.23 \\
\hline \multicolumn{4}{|l|}{ Fluids/transfusion (mL) } \\
\hline Saline & $1000(500,1500)$ & $1000(400,1800)$ & 0.84 \\
\hline Lactated Ringer's & $1700(1000,2000)$ & $1600(1200,2100)$ & 0.85 \\
\hline Cell saver & $400(300,450)$ & $250(300,430)$ & 0.49 \\
\hline Estimated blood loss (mL) & $200(123,500)$ & $200(123,500)$ & 0.79 \\
\hline Urine output (mL) & $350(265,600)$ & $515(325,710)$ & 0.13 \\
\hline
\end{tabular}

${ }^{a}$ Categorical variables are presented as count (frequency) and continuous variables as median (quartiles)

${ }^{\mathrm{b}}$ As estimated by the surgeon. Missing on seven patients lactate levels between groups $(2.1 \mathrm{mmol} / \mathrm{L}[1.6,3.3]$ vs. $2.0 \mathrm{mmol} / \mathrm{L}[1.7,2.3], p=0.43)$.

\section{Discussion}

In this phase II trial, we found no difference in postoperative lactate levels or clinical outcomes between patients receiving thiamine or placebo. We did find a significant difference in post-operative cellular and global oxygen consumption between the two groups.

To our knowledge, this is the second randomized, placebo-controlled study to date to examine the efficacy of thiamine in this patient population. Recently, Luger et al. enrolled 30 patients undergoing cardiac surgery [47]. Patients were randomized to one dose of preoperative thiamine $(300 \mathrm{mg})$ or placebo. Similar to our findings, they found no difference in post-operative lactate levels or clinical outcomes. There are a few key differences between the study by Luger et al. and that presented here. First, we enrolled more than twice the number of patients. Second, we only included moderateto high-risk patients (i.e., those with a EuroSCORE II > $1.5 \%)$. Third, we provided two doses of thiamine: one before and one after the surgery. Lastly, we included a number of additional outcomes including PDH measurements, global and cellular oxygen consumption, and more granular clinical outcomes including an assessment of post-operative complications. Despite these differences, the findings were largely similar.

Donnino et al. recently found that thiamine did not decrease 24-hour lactate levels as compared to placebo in the overall group of patients $(n=88)$ with septic shock and elevated lactate $(>3 \mathrm{mmol} / \mathrm{L})$. However, in those with

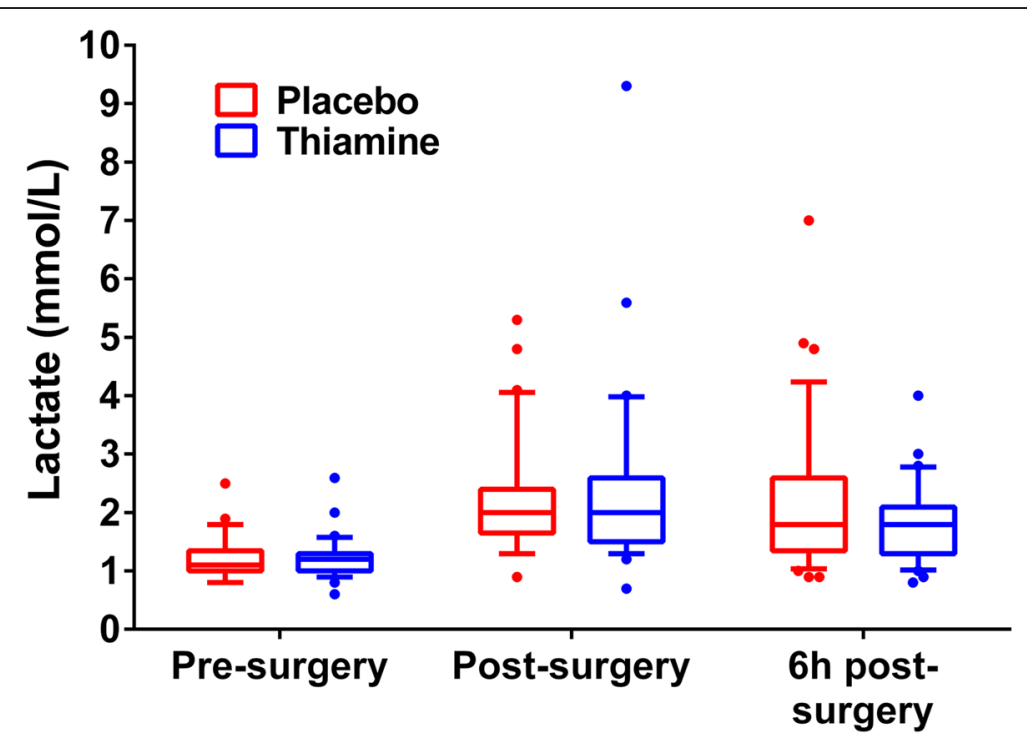

Fig. 3 Lactate levels over time between the two groups. There was no difference between the thiamine and placebo groups in the primary endpoint of lactate levels immediately after the surgery $(2.0[1.5,2.6] \mathrm{mmol} / \mathrm{L}$ vs. $2.0[1.7,2.4], p=0.75)$. The boxplots represent the $1^{\text {st }}$ quartiles, median, and $3^{\text {rd }}$ quartile. The whiskers represent the $10^{\text {th }}$ and $90^{\text {th }}$ percentile and outliers are marked with dots 

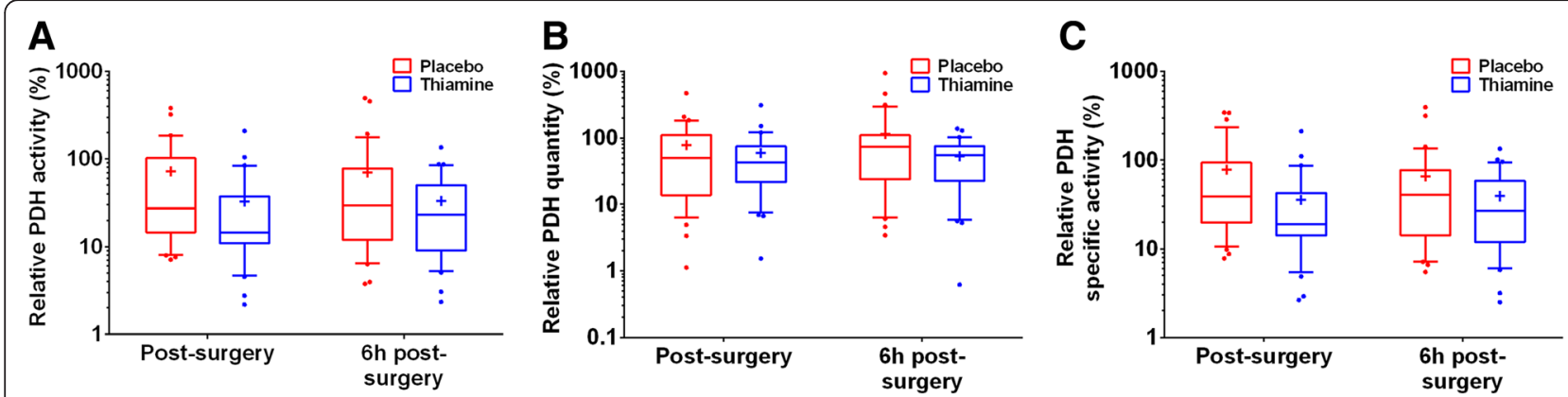

Fig. 4 PDH values. Relative PDH activity (a), quantity (b) and specific activity (c) post-surgery and 6 hours post-surgery. Values were calculated as relative to the pre-surgery level, which was set at $100 \%$. The $y$-axis is logarithmic to better illustrate the findings. The boxplots represent the $1^{\text {st }}$ quartiles, median, and $3^{\text {rd }}$ quartile. The whiskers represent the $10^{\text {th }}$ and $90^{\text {th }}$ percentile and outliers are marked with dots

baseline thiamine deficiency $(n=28)$, thiamine improved 24-hour lactate levels as compared to placebo and there was a signal toward decreased mortality in the thiamine group [48]. In the current study, only one patient was thiamine deficient at baseline and it is possible that thiamine administration only has clinical utility in this subgroup of patients. Alternatively, thiamine could be beneficial for the group that develops thiamine deficiency during surgery but there is no way to assess this subgroup in the current study since provision of thiamine in the study arm precludes the capacity to determine who would have become deficient.

We used post-operative lactate levels as the primary outcome for multiple reasons. First, if thiamine were to improve PDH activity, as hypothesized, pyruvate should be converted to acetyl-coenzyme A leading to increased oxygen utilization and decreased lactate levels. Second, multiple studies have found an association between elevated post-operative lactate levels and increased morbidity and mortality [5-10]. In a recent study, we found that post-operative lactate levels were associated with hospital and intensive care unit length of stay as well as post-operative non-surgical complications. This association remained after adjusting for more than 25 patient and surgical characteristics indicating that lactate could be a suitable surrogate for more patient-centered outcomes [10]. Lastly, as a continuous outcome, lactate levels are a reasonable outcome in a phase II trial from a statistical point of view. However, the post-operative lactate levels in the placebo group were lower than anticipated minimizing the potential for a treatment effect.

The finding that relative PDH activity was decreased in the thiamine group was unanticipated, remains largely unexplained, and could be a chance finding potentially due to baseline imbalances (i.e., a post hoc analysis accounting for baseline PDH activity showed no significant difference between groups). One challenge in measuring PDH activity includes replicating the in vivo

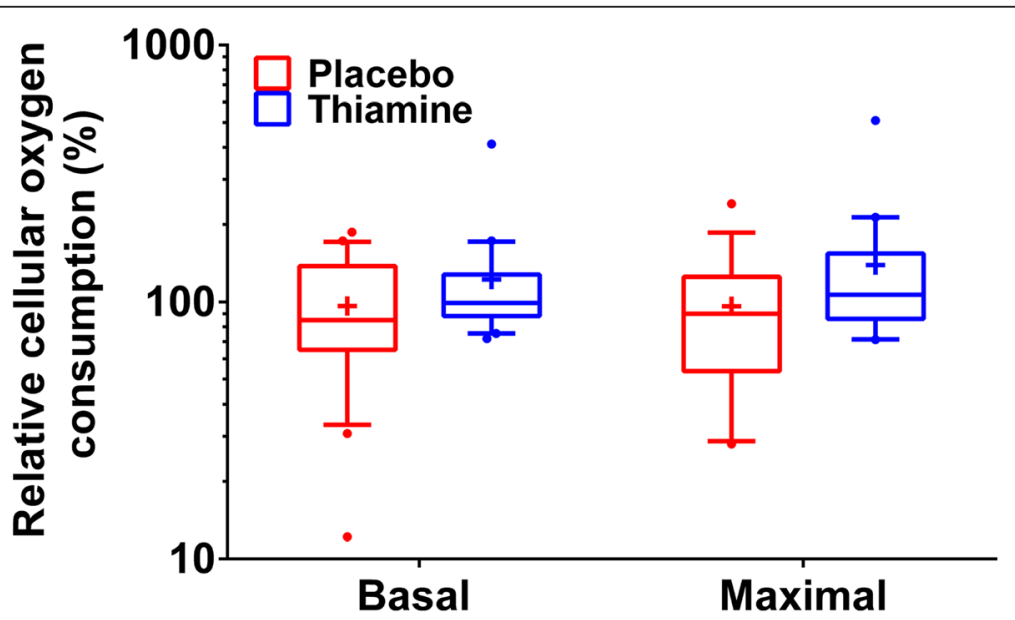

Fig. 5 Cellular oxygen consumption. We found a significant difference in post-surgery relative basal oxygen consumption between groups (99\% [89, $126]$ vs. $85 \%[66,136], p=0.04)$ and a significant difference in cellular maximal oxygen consumption between groups $(107 \%[86,155]$ vs. $90 \%[54$, 125], $p=0.02)$. The boxplots represent the $1^{\text {st }}$ quartiles, median, and $3^{\text {rd }}$ quartile. The whiskers represent the $10^{\text {th }}$ and $90^{\text {th }}$ percentile and outliers are marked with dots. The $y$-axis is logarithmic to better illustrate the findings 
Table 3 Post-operative complications

\begin{tabular}{llll}
\hline & $\begin{array}{l}\text { Thiamine } \\
(\mathrm{n}=31)\end{array}$ & $\begin{array}{l}\text { Placebo } \\
(\mathrm{n}=33)\end{array}$ & $p$ value \\
\hline Atrial fibrillation & $12(39)$ & $12(36)$ & 1.00 \\
Renal failure & $1(3)$ & $2(6)$ & 1.00 \\
Stroke & $0(0)$ & $0(0)$ & 1.00 \\
Myocardial infarction & $0(0)$ & $0(0)$ & 1.00 \\
Acute respiratory distress syndrome & $1(3)$ & $0(0)$ & 0.48 \\
Infection & $5(16)$ & $5(15)$ & 1.00 \\
Delirium & $3(10)$ & $4(12)$ & 1.00 \\
At least one complication & $16(52)$ & $16(48)$ & 1.00
\end{tabular}

environment. The PDH assay we used, which has been described in detail elsewhere [38], measures PDH activity in vitro in an "ideal" environment which includes the addition of a small amount of thiamine in order for the reaction to run. The provision of in vitro thiamine (even at small levels) limits the between-group comparison and might therefore not reflect in vivo $\mathrm{PDH}$ values. Future studies examining the administration of thiamine or other modulators of PDH might consider using measures of in vivo PDH activity although these methods are considerably more complex and might not be feasible in the CABG population [49].

We found that thiamine significantly improved global oxygen consumption after CABG surgery. For an average $80 \mathrm{~kg}$ patient the increase would be approximately $30 \mathrm{~mL} /$ min. In an open-label trial of thiamine administration in the critically ill, Berg et al. found that thiamine improved oxygen consumption [50], consistent with the findings reported here. We also found that cellular oxygen consumption was increased in the group receiving thiamine. We are not aware of any previous studies examining the effect of thiamine (or other clinical interventions) on cellular oxygen consumption. Although the improvements in cellular and global oxygen consumption did not result in improved clinical outcomes in the current trial, future studies should examine whether improvements in oxygen consumption could improve patient-centered outcomes particularly in diseases where ongoing impairment of oxygen consumption is believed to be pathologic and causative of organ injury. The fact that we found a difference between groups in oxygen consumption, but no difference in lactate levels may indicate that lactate elevation in this context is not solely related to oxygen consumption but could reflect other mechanisms such as decreased clearance or excessive adrenergic stimulation $[12,51]$. However, this remains speculative and will require additional studies.

The findings from the current study should be interpreted in the context of the study limitations. The sample size was relatively small and we might have been underpowered to detect differences between groups especially in the clinical outcomes. We only provided two doses of thiamine. While subsequent doses would not have affected our primary endpoint, they could affect more longterm outcomes. As noted above, the post-operative lactate levels were lower than expected and it is possible that including a more high-risk population with higher propensity for high post-operative lactate levels could have yielded different results. It is unknown whether measurements of $\mathrm{PDH}$ and oxygen consumption in PBMCs are representative of more relevant tissue such as the heart or the brain. Lastly, although the global oxygen consumption measurements used in this study has been validated [40, 41], this measurement is currently not considered the gold standard. The oxygen consumption results should be considered exploratory and hypothesis generating.

\section{Conclusions}

In this randomized, placebo-controlled, double-blind, phase II trial, we found no difference in post-operative lactate levels or clinical outcomes between patients receiving thiamine or placebo. We found a significant increase in post-operative oxygen consumption in patients receiving thiamine.

\section{Key messages}

- A randomized, double-blind, placebo-controlled trial of thiamine in 64 patients undergoing coronary artery bypass grafting was performed

- There was no difference in post-operative lactate levels between the two groups

- There was no difference in clinical outcomes between groups

- Patients in the thiamine groups had significantly higher post-operative cellular and global oxygen consumption

\section{Additional file}

Additional file 1: Supplemental methods and results. (DOCX 477 kb)

\section{Abbreviations}

CABG: Coronary artery bypass grafting; EuroSCORE: European System for Cardiac Operative Risk Evaluation; NYHA: New York Heart Association; OCR: Oxygen consumption rate; PBMC: Peripheral blood mononuclear cells; $\mathrm{PDH}$ : Pyruvate dehydrogenase; $\mathrm{VO}_{2}$ : Global oxygen consumption.

Competing interests

The authors declare that they have no competing interests.

\section{Authors' contributions}

LWA and MWD were responsible for study concept and design. LWA conducted the statistical analysis and drafted the manuscript. LWA, MJH, CS, JB, and $M M$ were responsible for acquisition of data. $X \mathrm{~L}$ and $S M$ were responsible for the laboratory work. LWA, MJH, KMB, MC, MNC, CS, JB, MM, SM, VS, DL, KK, $A L, V N, X L$, and MWD critically revised the manuscript for important intellectual content, interpreted the data, and approved the final version for submission. 


\section{Acknowledgments}

This study was funded by the American Heart Association (15CRP22830000) through a grant awarded to Dr. Andersen. Dr. Donnino was funded by the National Institutes of Heath/National Heart, Lung and Blood Institute (1K02HL107447-01A1), Dr. Berg by the American Heart Association (13CRP16930000), Dr. Chase by the National Institutes of Health/National Institute of General Medical Sciences (1K23GM101463-01 K1) and Dr. Cocchi by the American Heart Association (15SDG22420010). None of the funding sources were involved in the design and conduct of the study; collection, management, analysis, or interpretation of the data; preparation, review, or approval of the manuscript; or the decision to submit the manuscript for publication.

The authors would like to thank Francesca Montillo, M.M. for assisting with preparation of the manuscript, the preoperative staff and the nurses and respiratory therapists in the cardiovascular intensive care unit for assisting with study procedures, and Ana Urbin, Pharm.D. and Melissa Sciola, Pharm.D. for assisting with study drug preparation.

\section{Author details}

'Center for Resuscitation Science, Department of Emergency Medicine, Beth Israel Deaconess Medical Center, 330 Brookline Avenue, Boston, MA 02215, USA. ${ }^{2}$ Department of Anesthesiology, Aarhus University Hospital, Nørrebrogade 44, 8000 Aarhus C, Denmark. ${ }^{3}$ Research Center for Emergency Medicine, Aarhus University Hospital, Nørrebrogade 44, 8000 Aarhus C, Denmark. ${ }^{4}$ Department of Medicine, Division of Pulmonary and Critical Care, Beth Israel Deaconess Medical Center, 330 Brookline Avenue, Boston, MA 02215, USA. ${ }^{5}$ Department of Anesthesia Critical Care, Division of Critical Care, Beth Israel Deaconess Medical Center, 330 Brookline Avenue, Boston, MA 02215, USA. ${ }^{6}$ Department of Surgery, Division of Cardiothoracic Surgery, Beth Israel Deaconess Medical Center, 330 Brookline Avenue, Boston, MA 02215, USA. ${ }^{7}$ Department of Anesthesia, Beth Israel Deaconess Medical Center, 330 Brookline Avenue, Boston, MA 02215, USA. ${ }^{8}$ Clinical Research Center, Soroka University Medical Center, POB 151, Beer-Sheva 84965, Israel. ${ }^{9}$ Faculty of Health Sciences, Ben-Gurion University, POB 151, Beer-Sheva 84965, Israel.

\section{Received: 22 December 2015 Accepted: 16 February 2016} Published online: 14 March 2016

\section{References}

1. Hall MJ, DeFrances CJ, Williams SN, Golosinskiy A, Schwartzman A. National Hospital Discharge Survey: 2007 summary. Nat Health Stat Rep. 2010;29:1-20. 24.

2. Frost L, Molgaard H, Christiansen EH, Hjortholm K, Paulsen PK, Thomsen PE. Atrial fibrillation and flutter after coronary artery bypass surgery: epidemiology, risk factors and preventive trials. Int J Cardiol. 1992;36(3):253-61.

3. Saczynski JS, Marcantonio ER, Quach L, Fong TG, Gross A, Inouye SK, et al. Cognitive trajectories after postoperative delirium. N Engl J Med. 2012 367(1):30-9.

4. Silber JH, Rosenbaum PR, Schwartz JS, Ross RN, Williams SV. Evaluation of the complication rate as a measure of quality of care in coronary artery bypass graft surgery. JAMA. 1995:274(4):317-23.

5. Maillet JM, Le Besnerais $P$, Cantoni M, Nataf $P$, Ruffenach $A$, Lessana A, et al. Frequency, risk factors, and outcome of hyperlactatemia after cardiac surgery. Chest. 2003;123(5):1361-6.

6. Toraman F, Evrenkaya S, Yuce M, Aksoy N, Karabulut H, Bozkulak Y, et al. Lactic acidosis after cardiac surgery is associated with adverse outcome. Heart Surg Forum. 2004;7(2):E155-9.

7. Hajjar LA, Almeida JP, Fukushima JT, Rhodes A, Vincent JL, Osawa EA, et al. High lactate levels are predictors of major complications after cardiac surgery. J Thorac Cardiovasc Surg. 2013;146(2):455-60.

8. Lindsay AJ, Xu M, Sessler DI, Blackstone EH, Bashour CA. Lactate clearance time and concentration linked to morbidity and death in cardiac surgical patients. Ann Thorac Surg. 2013;95(2):486-92.

9. Badreldin AM, Doerr F, Elsobky S, Brehm BR, Abul-dahab M, Lehmann T, et al. Mortality prediction after cardiac surgery: blood lactate is indispensible. Thorac Cardiovasc Surg. 2013;61(8):708-17.

10. Andersen LW, Holmberg MJ, Doherty M, Khabbaz K, Lerner A, Berg KM, et al. Postoperative lactate levels and hospital length of stay after cardiac surgery. J Cardiothorac Vasc Anesth. 2015;29:1454-60.

11. Casserly B, Phillips GS, Schorr C, Dellinger RP, Townsend SR, Osborn TM, et al. Lactate measurements in sepsis-induced tissue hypoperfusion: results from the Surviving Sepsis Campaign database. Crit Care Med. 2015;43(3):567-73.
12. Andersen LW, Mackenhauer J, Roberts JC, Berg KM, Cocchi MN, Donnino MW. Etiology and therapeutic approach to elevated lactate levels. Mayo Clin Proc. 2013;88(10):1127-40.

13. Fink MP. Bench-to-bedside review: cytopathic hypoxia. Crit Care. 2002;6(6):491-9.

14. Shoemaker WC, Appel PL, Kram HB, Waxman K, Lee TS. Prospective trial of supranormal values of survivors as therapeutic goals in high-risk surgical patients. Chest. 1988;94(6):1176-86.

15. Rivers EP, Rady MY, Martin GB, Fenn NM, Smithline HA, Alexander ME, et al. Venous hyperoxia after cardiac arrest. Characterization of a defect in systemic oxygen utilization. Chest. 1992;102(6):1787-93.

16. Hayes MA, Timmins AC, Yau EH, Palazzo M, Hinds CJ, Watson D. Elevation of systemic oxygen delivery in the treatment of critically ill patients. N Engl J Med. 1994;330(24):1717-22.

17. Patel MS, Korotchkina LG. Regulation of the pyruvate dehydrogenase complex. Biochem Soc Trans. 2006;34(Pt 2):217-22.

18. Linn TC, Pettit FH, Reed LJ. Alpha-keto acid dehydrogenase complexes. X. Regulation of the activity of the pyruvate dehydrogenase complex from beef kidney mitochondria by phosphorylation and dephosphorylation. Proc Natl Acad Sci U S A. 1969;62(1):234-41.

19. Andersen LW, Liu X, Peng TJ, Giberson TA, Khabbaz KR, Donnino MW. Pyruvate dehydrogenase activity and quantity decreases after coronary artery bypass grafting: a prospective observational study. Shock. 2015;43(3):250-4.

20. Kobayashi K, Neely JR. Effects of ischemia and reperfusion on pyruvate dehydrogenase activity in isolated rat hearts. J Mol Cell Cardiol. 1983;15(6):359-67.

21. Patel TB, Olson MS. Regulation of pyruvate dehydrogenase complex in ischemic rat heart. Am J Physiol. 1984;246(6 Pt 2):H858-64.

22. Lewandowski ED, Johnston DL. Reduced substrate oxidation in postischemic myocardium: 13C and 31P NMR analyses. Am J Physiol. 1990:258(5 Pt 2):H1357-65.

23. Rao V, Merante F, Weisel RD, Shirai T, Ikonomidis JS, Cohen G, et al. Insulin stimulates pyruvate dehydrogenase and protects human ventricular cardiomyocytes from simulated ischemia. J Thorac Cardiovasc Surg. 1998:116(3):485-94.

24. Merante F, Mickle DA, Weisel RD, Li RK, Tumiati LC, Rao V, et al. Myocardial aerobic metabolism is impaired in a cell culture model of cyanotic heart disease. Am J Physiol. 1998;275(5 Pt 2):H1673-81.

25. Naito E, Ito M, Yokota I, Saijo T, Matsuda J, Kuroda Y. Thiamine-responsive lactic acidaemia: role of pyruvate dehydrogenase complex. Eur J Pediatr. 1998;157(8):648-52.

26. Donnino MW, Cocchi MN, Smithline H, Carney E, Chou PP, Salciccioli J. Coronary artery bypass graft surgery depletes plasma thiamine levels. Nutrition. 2010:26(1):133-6.

27. Society of Thoracic Surgeons National Database - Data Collection. Available from: http://www.sts.org/sts-national-database/database-managers/adultcardiac-surgery-database/data-collection. Accessed date Dec 222015.

28. Sacco RL, Kasner SE, Broderick JP, Caplan LR, Connors JJ, Culebras A, et al. An updated definition of stroke for the 21st century: a statement for healthcare professionals from the American Heart Association/American Stroke Association. Stroke. 2013;44(7):2064-89.

29. Thygesen K, Alpert JS, Jaffe AS, Simoons ML, Chaitman BR, White HD, et al. Third universal definition of myocardial infarction. Circulation. 2012;126(16):2020-35.

30. Force ADT, Ranieri VM, Rubenfeld GD, Thompson BT, Ferguson ND, Caldwell E, et al. Acute respiratory distress syndrome: the Berlin Definition. JAMA. 2012; 307(23):2526-33.

31. The Criteria Committee of the New York Heart Association. Nomenclature and criteria for diagnosis of diseases of the heart and great vessels. 9th ed. Boston, Mass: Little, Brown \& Co; 1994.

32. Campeau L. Letter: grading of angina pectoris. Circulation. 1976;54(3):522-3.

33. EuroSCORE II calculator. Available from: http://www.euroscore.org/calc.html. Accessed date Dec 222015.

34. Nashef SA, Roques F, Michel P, Gauducheau E, Lemeshow S, Salamon R. European system for cardiac operative risk evaluation (EuroSCORE). Eur J Cardiothorac Surg. 1999;16(1):9-13.

35. Carnero-Alcazar M, Silva Guisasola JA, Reguillo Lacruz FJ, Maroto Castellanos LC, Cobiella Carnicer J, Villagran Medinilla E, et al. Validation of EuroSCORE II on a single-centre 3800 patient cohort. Interact Cardiovasc Thorac Surg. 2013;16(3):293-300.

36. Biancari F, Vasques F, Mikkola R, Martin M, Lahtinen J, Heikkinen J. Validation of EuroSCORE II in patients undergoing coronary artery bypass surgery. Ann Thorac Surg. 2012;93(6):1930-5. 
37. Harris PA, Taylor R, Thielke R, Payne J, Gonzalez N, Conde JG. Research electronic data capture (REDCap)-a metadata-driven methodology and workflow process for providing translational research informatics support. J Biomed Inform. 2009;42(2):377-81.

38. Liu X, Pervez H, Andersen LW, Uber A, Montissol S, Patel P, et al. Immunocapture and microplate-based activity and quantity measurement of pyruvate dehydrogenase in human peripheral blood mononuclear cells. Bioanalysis. 2015;7(5):583-92.

39. Lib M, Rodriguez-Mari A, Marusich MF, Capaldi RA. Immunocapture and microplate-based activity measurement of mammalian pyruvate dehydrogenase complex. Anal Biochem. 2003;314(1):121-7.

40. McLellan S, Walsh T, Burdess A, Lee A. Comparison between the DatexOhmeda M-COVX metabolic monitor and the Deltatrac II in mechanically ventilated patients. Intensive Care Med. 2002;28(7):870-6.

41. Donaldson L, Dodds S, Walsh TS. Clinical evaluation of a continuous oxygen consumption monitor in mechanically ventilated patients. Anaesthesia. 2003:58(5):455-60.

42. Ferrick DA, Neilson A, Beeson C. Advances in measuring cellular bioenergetics using extracellular flux. Drug Discov Today. 2008;13(5-6):268-74.

43. Kahan BC, Morris TP. Reporting and analysis of trials using stratified randomisation in leading medical journals: review and reanalysis. BMJ. 2012;345:e5840.

44. Thornalley PJ, Babaei-Jadidi R, Al Ali H, Rabbani N, Antonysunil A, Larkin J, et al. High prevalence of low plasma thiamine concentration in diabetes linked to a marker of vascular disease. Diabetologia. 2007;50(10):2164-70.

45. Larkin JR, Zhang F, Godfrey L, Molostvov G, Zehnder D, Rabbani N, et al. Glucose-induced down regulation of thiamine transporters in the kidney proximal tubular epithelium produces thiamine insufficiency in diabetes. PLoS One. 2012;7(12):e53175.

46. Fergusson D, Aaron SD, Guyatt G, Hebert P. Post-randomisation exclusions: the intention to treat principle and excluding patients from analysis. BMJ. 2002;325(7365):652-4.

47. Luger M, Hiesmayr M, Koppel P, Sima B, Ranz I, Weiss C, et al. Influence of intravenous thiamine supplementation on blood lactate concentration prior to cardiac surgery: a double-blinded, randomised controlled pilot study. Eur J Anaesthesiol. 2015;32(8):543-8.

48. Donnino MW, Andersen LW, Chase M, Berg KM, Tidswell M, Giberson T, et al. Randomized, double-blind, placebo-controlled trial of thiamine as a metabolic resuscitator in septic shock: a pilot study. Crit Care Med. 2016;44(2):360-7.

49. Atherton HJ, Schroeder MA, Dodd MS, Heather LC, Carter EE, Cochlin LE, et al. Validation of the in vivo assessment of pyruvate dehydrogenase activity using hyperpolarised 13C MRS. NMR Biomed. 2011;24(2):201-8

50. Berg KM, Gautam S, Salciccioli JD, Giberson T, Saindon B, Donnino MW. Intravenous thiamine is associated with increased oxygen consumption in critically ill patients with preserved cardiac index. Ann Am Thorac Soc 2014;11(10):1597-601.

51. Garcia-Alvarez M, Marik P, Bellomo R. Sepsis-associated hyperlactatemia. Crit Care. 2014;18(5):503

\section{Submit your next manuscript to BioMed Central and we will help you at every step:}

- We accept pre-submission inquiries

- Our selector tool helps you to find the most relevant journal

- We provide round the clock customer support

- Convenient online submission

- Thorough peer review

- Inclusion in PubMed and all major indexing services

- Maximum visibility for your research

Submit your manuscript at www.biomedcentral.com/submit

) Biomed Central 